\title{
How do I select the right clinical teaching fellowship?
}

There are many considerations to weigh up when considering a clinical teaching fellowship, Abi Rimmer hears

\section{Abi Rimmer}

The BMJ

\section{Avoid posts without a clear structure}

Phil Walmsley, consultant orthopaedic surgeon, NHS Fife, says, "Clinical teaching is exceptionally rewarding, and the opportunity to take up a post that allows you to concentrate on it can be beneficial, both professionally and personally. Over recent years the number of posts has expanded rapidly, but they vary in quality and opportunity. This variation lets candidates find a post that suits their needs.

"The majority of posts will have a formal teaching commitment. But what proportion of your work this will be will differ, as will post length, staff levels, environment, clinical involvement, and involvement in developing teaching material for university courses.

"Before applying for a fellowship it's worth contacting those who've completed it to get a clearer understanding of what it entails. To make an informed choice, you need to establish why you want to undertake the post.

"Things to consider include the stage you are at in your career, the length of the post, and how it fits into your career plan. You should also think about how you want to develop your skills as a clinical teacher, the availability of mentoring during the post, and what access is available to a formal teaching qualification.

"If you can, avoid posts with a heavy clinical or on-call commitment and those with supervisors who have no clear interest in teaching. You should also be wary of posts without a structured teaching programme and those which are essentially ill-defined research posts, unless you are planning full time educational research."

\section{Consider the clinical and teaching split}

Shamim Nassrally, chief registrar and acute internal medicine specialty trainee year 7, says, "Teaching fellowships are an invaluable opportunity to develop clinical teaching skills and can help you gain experience in leadership, management, and educational theory. There are a number of things to consider when applying. Consider whether the role is mainly clinical skills focused, classroom based, or simulation based, and what the distribution is between the different educational modalities if the role is a mixture of these methods.

"You should also consider how your time would be split between clinical and teaching activities. Some fellowships offer one day a week or more to continue your clinical work which can be important for craft based specialties that might need continuing practice to maintain skills.

"You should also find out if a formal educational qualification is part of the package. Many fellowships fund fellows to study towards an educational qualification such as a postgraduate award or diploma in medical education. Is this the case and, if it is, is the time allocated for an educational qualification part of the role?

"It's also important to know whether the role includes on-call commitments. Time away may let you focus on your educational role, and give you some respite from antisocial clinical commitments and a year without night shifts. A teaching fellowship, along with many similar roles, can help reduce the burnout associated with high intensity clinical work."

\section{Speak to previous fellows}

Oluwaseun Anyiam, specialty trainee year 5 in diabetes and endocrinology and National Institute for Health Research academic clinical fellow, says, "The most important factor to consider when choosing a clinical teaching fellowship is the proportion of dedicated teaching time it offers. This can range from $20 \%$ to $100 \%$ and should be detailed in the job specification.

"Some people prefer a higher teaching load which may come with additional responsibilities, such as organisation of teaching. Others may prefer a greater clinical component to improve their understanding of a particular specialty. I suggest speaking to previous fellows to get an idea of how the split works in practice.

"The specialty of the clinical component is another important consideration. Clinical teaching fellowships are great opportunities to learn more about a specialty of interest to you. Working within the specialty can provide valuable experience, particularly if you are undecided about what to commit to long term.

"It's also worth thinking about the hospital in which the fellowship is based. Tertiary centres are generally linked to universities and tend to have more medical students, although district general hospitals can have large numbers of students too. Moreover, a hospital having fewer students is not necessarily a negative as this can allow for closer student-tutor relationships and the potential for peer-mentorship roles during the fellowship. 
"Remember that clinical teaching fellowships are rewarding and fulfilling experiences and will provide you with skills to take forward into the rest of your career."

\section{Some fellowships fund qualifications}

Roberta Morris, clinical teaching fellow at Newcastle University Medicine Malaysia, says, "As a foundation year 2 doctor, I decided not to go directly into specialty training-this left me with a dilemma over whether to become a clinical teaching fellow or work abroad. Luckily, I found my current post in Malaysia, where I am gaining teaching experience while being exposed to the healthcare and culture of another country, with the opportunity to travel.

"You can find six month, one year, and two year fellowship posts if you are willing to be flexible. My current post is for two years; the first year is $100 \%$ teaching and the second year is negotiable in terms of the amount of specialty, clinical, and teaching work I will do. Funding for qualifications up to the postgraduate certificate in medical education should now be standard in most one year programmes, but if you take a two year post you can expect to be funded through the postgraduate diploma with an option to self fund up to masters level.

"When looking at a post, consider which years of the medical degree programme you want to teach. I am involved in teaching all five years which has given me experience in delivering a wide range of material, from basic science lectures to final year simulation.

"Also consider the size of the institution you will be working at. At smaller institutions, junior staff can be actively involved in creating new materials, curriculum changes, and research while developing specialist interests. A larger institution, however, may offer more specialised clinical or research opportunities." 